\title{
Técnicas cromatográficas e o desafio na determinação de compostos presentes em matrizes complexas: Relato de um caso prático do produto natural piperlongumina
}

\author{
Lucas Maciel Mauriz Marques', Anderson Rodrigo Moraes de Oliveira²* \\ ${ }^{1}$ Departamento de Física-Química, Faculdade de Ciências Farmacêuticas, Universidade de São Paulo - USP, \\ Cep 14040-903, Ribeirão Preto, SP, Brasil \\ ${ }^{2}$ Departamento de Química, Faculdade de Filosofia, Ciências e Letras, Universidade de São Paulo - USP, \\ Cep 14040-901, Ribeirão Preto, SP, Brasil \\ e-mail: deoliveira@usp.br
}

Resumo

A elevada diversidade química dos produtos naturais de plantas representa um desafio para os métodos analíticos de separação e detecção, sendo difícil empregar um procedimento único de análise para todos os compostos presentes em um extrato. Atualmente, métodos cromatográficos como a CLAE, a CLUE e a CG têm desempenhado um importante papel quanto à eficiência de separação. A vantagem de cada uma dessas técnicas dependerá do propósito experimental requerido. No presente trabalho analisar-se-ão as vantagens e desvantagens de cada uma dessas técnicas cromatográficas na análise do(s) metabólitos(s) do produto natural piperlongumina, após essa ser metabolizada por microssomas de fígado de ratos. Mediante as comparações, conclui-se que as técnicas não se excluem e não são competitivas, pelo contrário, são complementares, cada uma com suas especificidades e limitações.

Palavras-chave

CLAE; CLUE; CG; produto natural.

\section{Chromatographic techniques and the challenge for the determination of compounds in complex matrices: Report of a case study of the natural product piperlongumine}

\section{Abstract}

The chemical diversity of the natural products from plants represents a challenge for analytical methods of separation and detection. It is difficult to employ a single procedure to analise all compounds. Currently, chromatographic methods such as HPLC, GC and UHPLC have played an important role on the separation efficiency. The advantage arising from each one will depend on the experimental requirements. In this study, it will be analyzed the advantages and disavantages of these chromatographic techniques for determination of piperlongumine, as well as the resulting metabolites from the in vitro metabolism performed by rat liver microsomes. Regarding to the comparison, it is concluded that the techniques do not compete with them, unlike; they are complementary to each other.

Keywords

HPLC; UHPLC; GC; natural products. 


\section{Introdução}

\subsection{Produtos naturais e técnicas cromatográficas}

Desde o início da história da humanidade, os produtos naturais (PN) sempre foram investigados e utilizados para tratamento de inúmeras doenças, sendo fonte para o desenvolvimento da maioria dos medicamentos utilizados ${ }^{[1]}$. A razão para isso pode ser explicada pela elevada diversidade química, a qual representa um desafio para os métodos analíticos de separação e detecção ${ }^{[2]}$, sendo difícil empregar um procedimento único de análise para todos os compostos, levando-se em conta que os analitos podem estar presentes em pequenas ou grandes quantidades e, dependendo do tipo de estudo (quantificação, fingerprinting, análise de traços etc.), requerem-se elevada sensibilidade e seletividade para a sua deteç̧ão ${ }^{[3,4]}$.

Ao longo dos últimos 100 anos, a cromatografia se desenvolveu a partir de uma ferramenta rudimentar para a separação dos pigmentos em um conjunto de técnicas capazes de enfrentar os mais complexos problemas de análise, separação e purificação dos componentes de uma amostra. No decurso do seu crescimento, podem-se destacar basicamente três importantes referências: a introdução da cromatografia, a contribuição dos estudos de Martin, na década de 1950, e a introdução do equipamento de cromatografia líquida de alta eficiência (CLAE), na década de $1970^{[5]}$.

Até o advento da CLAE, as separações de compostos, principalmente os oriundos de plantas, eram realizados por cromatografia em coluna aberta, em papel ou em camada delgada. Suas desvantagens: a grande quantidade de amostra requerida e o tempo gasto no caso da cromatografia em coluna aberta, o que foi melhorado com o emprego de menores quantidades de amostra na cromatografia em papel e em camada delgada; no entanto, a quantificação era ainda inadequada e havia dificuldade na resolução de compostos similares ${ }^{[5]}$.

Atualmente, métodos cromatográficos como a CLAE, a CLUE (cromatografia líquida de ultra eficiência) e a CG (cromatografia gasosa) têm desempenhado importante papel quanto à eficiência de separação ${ }^{[5-7]}$. A vantagem advinda de cada um deles dependerá do propósito experimental requerido ${ }^{[8]}$.

\subsection{CLAE, CLUE e CG}

A análise de PN exige métodos de separação eficientes antes da sua detecção. A CLAE tem sido reconhecida desde o início da década de 1980 como a técnica mais versátil para esse fim. Ao longo dos anos, ela vem passando por aprimoramentos em termos de velocidade de análise, tipos de fases estacionárias empregadas, detectabilidade, aplicabilidade a uma variedade de amostras, além da capacidade de hifenação com detectores espectrométricos. Do ponto de vista cromatográfico, colunas com diferentes fases vêm permitindo uma melhor separação de compostos e o desenvolvimento mais recente inclui o emprego de colunas estáveis em uma maior faixa de $\mathrm{pH}$, contendo partículas com tamanho menores que $5 \mu \mathrm{m}^{[4,9]}$. Isso porque as propriedades químicas do material de enchimento e as propriedades da coluna necessitam ser levadas em consideração ${ }^{[4]}$. Muitas vezes, o uso de $\mathrm{pH}$ elevado é necessário a fim de se evitar, por exemplo, a coeluição de picos ou o aparecimento de picos assimétricos.

Essas tendências na cromatografia líquida estão permitindo uma adequada seletividade e redução no tempo de análise sem comprometer a qualidade da separação, gerando picos com resolução aceitável. Desse modo, em 2004 foi lançado o primeiro sistema de CLUE disponível 
no mercado (Waters Corporation), sendo possível encontrar vários nomes para essa técnica: resolução rápida, separação rápida e separação ultrarrápida ${ }^{[10]}$.

A CLUE é uma ferramenta de separação rápida ideal para análise de amostras complexas em ambos os modos: gradiente e isocrático. Em comparação com a CLAE, a eficiência é maior, com menor alargamento de banda, maiores valores de resolução, maior velocidade de análise e melhor detectabilidade por meio da utilização de partículas de sub- $2 \mu \mathrm{m}$, o que gera picos mais finos, melhorando assim a relação sinal/ruído ${ }^{[11]}$. Essa redução no tamanho das partículas resultou, segundo o modelo teórico descrito por van Deemter, na diminuição dos caminhos múltiplos e da resistência à transferência de massa, fatores esses responsáveis pelo alargamento de bandas em CLAE. Embora a CLAE apresente algumas vantagens relacionadas à especificidade e detectabilidade, o tempo necessário para análise é relativamente longo, de $20 \mathrm{~min}$ a $80 \mathrm{~min}$. A CLUE, por sua vez, o reduz marcadamente em até 20 vezes, sem que isto comprometa a resolução ${ }^{[12-14]}$. Um benefício adicional da CLUE é o baixo consumo de fase móvel ${ }^{[10]}$.

A alteração deu-se não somente no tamanho, mas foram desenvolvidas partículas híbridas com pontes de etileno (Acquity BEH - Waters Corporation), obtidas por meio da cocondensação de 1,2-bis-(trietoxissilil)-etano (BTE) com tetraetoxissilano (TEOS). Com a presença dessas pontes, a maioria dos grupos silanóis livres está protegida $^{[15]}$, conferindo elevada estabilidade química ao longo de quase toda a faixa de $\mathrm{pH}$ (1 a 12), diminuição de interações secundárias indesejáveis com esses grupamentos ${ }^{[10]}$ e maior estabilidade mecânica, por meio das ligações silício-carbono, que garante resistência a altas pressões $^{[11]}$.
Além da cromatografia líquida, emprega-se também a cromatografia gasosa (CG) na análise de PN. Sessenta anos se passaram desde que o seu princípio foi demonstrado pela primeira vez por James e Martin ${ }^{[16]}$. É uma técnica analítica bem estabelecida, comumente utilizada para a caracterização e identificação de compostos voláteis. Possui excelente poder de resolução, tornando possível, muitas vezes, a análise de dezenas de substâncias de uma mesma amostra ${ }^{[17,18]}$. A restrição para análise de amostras voláteis (menos de $20 \%$ dos compostos orgânicos podem ser separados) faz com que a cromatografia líquida seja uma técnica alternativa para separação de compostos não voláteis e termolábeis ${ }^{5}$.

No presente trabalho serão discutidas as vantagens e desvantagens de cada uma dessas técnicas cromatográficas no desenvolvimento de um método para análise do(s) metabólitos(s) do produto natural piperlongumina (Figura 1), obtido após metabolismo por microssomas de fígado de ratos.

\section{Parte experimental}

\subsection{Reagentes e solventes}

Acetato de etila, acetonitrila (ACN) e metanol grau HPLC foram obtidos da J.T. Baker (Phillipsburg, EUA). D-Glicose 6-fosfato monossódico, glicose 6-fosfato desidrogenase

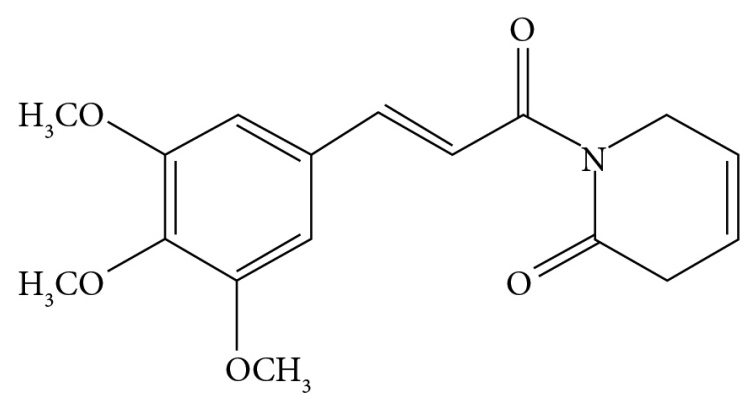

Figura 1 Estrutura química da piperlongumina. 
tipo VII, $\beta$-nicotinamida adenina dinucleotídeo foram obtidos da Sigma-Aldrich (Darmstadt, Alemanha). Fosfato de sódio monobásico anidro PA foi comprado da Synth (SP, Brasil). Hepes, Tris (base) foram obtidos da J.T. Baker (Phillipsburg, EUA). A piperlongumina (isolada de Piper tuberculatum) foi gentilmente cedida pelo prof. dr. Massuo Jorge Kato, do IQ-USP.

\subsection{2 .2 Análises cromatográficas}

\subsubsection{CLAE-DAD ${ }^{[19]}$}

Equipamento de CLAE marca Shimadzu (Kioto, Japão), composto de uma bomba LC-20AT, operando a uma vazão de $1 \mathrm{~mL} \mathrm{~min}^{-1}$, degaseificador DGU-20A, injetor com amostrador de $50 \mu \mathrm{L}$ SIL-10AF, detector por arranjo de diodo SPD-M20A (190 nm a $800 \mathrm{~nm}$ ), controladora CBM-20A e forno para coluna modelo CTO-20A, temperatura de análise de $32{ }^{\circ} \mathrm{C}$. As injeções foram realizadas automaticamente $(20 \mu \mathrm{L})$ e o programa empregado na aquisição dos dados foi o software LC solution, SPDM20A PDA utility (Shimadzu', Kioto, Japão). Utilizou-se a coluna cromatográfica Shim-pack VP-ODS Shimadzu $(250 \mathrm{~mm} \times 4,6 \mathrm{~mm}$ d.i., $4,6 \mu \mathrm{m})$ e coluna de guarda Shim-pack GVPODS Shimadzu $(10 \mathrm{~mm} \times 4,6 \mathrm{~mm}$ d.i., 4,6 $\mu \mathrm{m})$ (Kioto, Japão). Como fase móvel, foi utilizada acetonitrila $40 \%$ e água $60 \%(\mathrm{v} / \mathrm{v})$ para o modo de eluição isocrático. Para o modo por gradiente foi utilizado acetonitrila e água, ambos com $1 \%$ de ácido acético (v/v), que iniciava com $10 \%$ de ACN e atingia 100\% em 30 minutos, a uma vazão de $1 \mathrm{~mL} \mathrm{~min}^{-1}$.

\subsubsection{CLUE-DAD ${ }^{[19]}$}

Equipamento de CLUE: Acquity UPLC $^{\mathrm{rm}}$ DAD da Waters Corporation (Milford, Massachusetts, EUA), coluna Acquity ${ }^{\mathrm{rm}}$ UPLC $\mathrm{BEH}$ C18 (Bridged Ethylsiloxane Hybrid)
(50 $\mathrm{mm} \times 2,1 \mathrm{~mm}, 1,7 \mu \mathrm{m})$ e temperatura de análise de $32{ }^{\circ} \mathrm{C}$. Como fase móvel, foi utilizado modo de eluição por gradiente, empregando acetonitrila e água, ambas com $1 \%$ de ácido acético (v/v), que iniciava com $10 \%$ de $\mathrm{ACN}$ e atingia $100 \%$ em 4 minutos. A vazão empregada foi de $0,3 \mathrm{~mL} \mathrm{~min}{ }^{-1}$ e o volume de injeção de $5 \mu \mathrm{L}$.

\subsubsection{CG-EM}

Empregou-se um cromatógrafo CG-EM QP2010 série C704643 equipado com autoinjetor AOC-5000, ambos da Shimadzu (Kioto, Japão). Foi utilizada coluna capilar de sílica fundida, DB-1MS $(30 \mathrm{~m} \times 0,25 \mathrm{~mm}, 0,25 \mu \mathrm{m}$, Agilent Technologies, J \& W Scientific, Folsom, CA, EUA). Um $\mu \mathrm{L}$ foi injetado no modo splitless com temperatura de injeção de $250^{\circ} \mathrm{C}$ e o intervalo de massas analisadas foi de $\mathrm{m} / \mathrm{z}$ 40-400, energia de ionização de $70 \mathrm{eV}$. As análises das amostras teste foram feitas no modo full scan, sendo empregado hélio como gás de arraste, a uma velocidade linear de $42,9 \mathrm{~cm} \mathrm{~s}^{-1}$. A temperatura da fonte de íons e da interface foi de $250{ }^{\circ} \mathrm{C}$ e $280^{\circ} \mathrm{C}$, respectivamente. A temperatura inicial de análise foi de $100{ }^{\circ} \mathrm{C}$, sendo mantida durante cinco minutos. Após, a temperatura foi aumentada a $5{ }^{\circ} \mathrm{C} \mathrm{min}{ }^{-1}$ até atingir $290^{\circ} \mathrm{C}$, assim permanecendo por sete minutos.

\subsection{Procedimento de incubação para o estudo de metabolismo in vitro e preparo das amostras $[19,20]$}

A mistura de incubação (1 mL) consistiu de: $725 \mu \mathrm{L}$ de tampão fosfato de sódio $0,25 \mathrm{~mol}$ $\mathrm{L}^{-1}(\mathrm{pH} 7,4), 250 \mu \mathrm{L}$ da solução de incubação contendo $\mathrm{NADP}^{+}$(fosfato de dinucleótido de nicotinamida e adenina) (0,25 $\mathrm{mM})$, glicose-6-fosfato (5 mM) e glicose-6-fosfato desidrogenase $\left(0,5\right.$ unidades $\left.\mathrm{mL}^{-1}\right)$ em tampão Tris- $\mathrm{HCl}$ $0,05 \mathrm{~mol} \mathrm{~L}^{-1}\left(\mathrm{pH} \mathrm{7,4)}\right.$ com 0,15 mol L-1 $\mathrm{KCl}^{[20]}$. Adicionou-se a fração microssomal do fígado de rato (Protocolo CEUA n. 11.1.1047.53.4), a 
fim de obter-se uma concentração protéica de $1 \mathrm{mg} \mathrm{mL} \mathrm{mL}^{-1}$. Esse meio foi fortificado com $25 \mu \mathrm{L}$ das soluções padrão da piperlongumina (concentração alta $1 \mathrm{mg} \mathrm{mL} \mathrm{m}^{-1}$ e baixa $\left.250 \mu \mathrm{g} \mathrm{mL} \mathrm{mL}^{-1}\right) \mathrm{e}$ o procedimento de metabolismo in vitro iniciou-se. A incubação foi realizada sob agitação a 37 ${ }^{\circ} \mathrm{C}$, em banho-maria, modelo SL 157 (Solab, SP, Brasil), durante 50 minutos. A reação foi encerrada ao adicionarem-se $4 \mathrm{~mL}$ de solvente orgânico extrator (acetato de etila); em seguida as amostras foram agitadas por 15 minutos a $1.000 \mathrm{rpm}$ (modelo VRX, IKA`, Stafuen, Alemanha). Após essa etapa, centrifugaram-se os tubos a $2.000 \mathrm{~g}$ durante cinco minutos a $5{ }^{\circ} \mathrm{C}$ (modelo Himac CF16RXII, Hitachi, Tóquio, Japão). Retiraram-se $3 \mathrm{~mL}$ de solvente orgânico, e transferidos para outro tubo. Por fim, as amostras foram evaporadas até secura sob fluxo de ar comprimido. Os resíduos foram ressuspensos em $100 \mu \mathrm{L}$ de fase móvel e levados para análise. Amostras controles foram preparadas contendo microssomas inativos e sem o sistema de regeneração de NADPH. Amostras branco também foram preparadas sem adição da piperlongumina. Ambas foram submetidas ao mesmo procedimento de incubação anteriormente descrito.

\section{Resultados e discussão}

Inicialmente desenvolveu-se um método de análise no modo isocrático em equipamento de CLAE-DAD, para detecção da piperlongumina e os possíveis produtos formados no metabolismo in vitro e, assim, verificação do perfil do meio reacional. Levando-se em conta que os fatores responsáveis pela alteração do tempo de retenção de um composto são composição da fase móvel, afinidade do analito pela fase estacionária e tipo de eluição empregada ${ }^{[10]}$, diferentes proporções entre os solventes foram utilizadas: ACN: $\mathrm{H}_{2} \mathrm{O}$ 40: 60, 50: 50, 60: 40 e 70: 30 (v/v), a fim de obterem-se resolução aceitável, picos simétricos e curto tempo de análise. A proporção escolhida foi ACN: $\mathrm{H}_{2} \mathrm{O}(40: 60 \mathrm{v} / \mathrm{v})$. Conforme apresentado na Figura 2, há a presença de dois picos, bem resolvidos e simétricos, um relacionado à piperlongumina em $t_{R}=15,41 \mathrm{~min}$, o qual também apareceu na amostra controle, e o outro em $t_{R}=7,12 \mathrm{~min}$, relacionado ao produto do metabolismo in vitro, que não apareceu na amostra controle. Por questão de detectabilidade dos compostos, injetou-se uma solução de concentração $1 \mathrm{mg} \mathrm{mL}^{-1}$ obtida a partir de um pool de 30 replicatas, uma vez que os detectores ópticos de CLAE (UV - ultravioleta e DAD - arranjo de diodos) são projetados para suportar injeções de grandes quantidades de amostra, por longos períodos de análise, sem que isso cause sua saturação ${ }^{14}$.

Com o emprego dessa condição, o tempo total da análise foi de 18 minutos; entretanto, com o intuito de desenvolver um método mais rápido, visando os diversos ensaios futuros que exigiriam muitas replicatas, e também a economia de fase móvel, partiu-se para o desenvolvi-

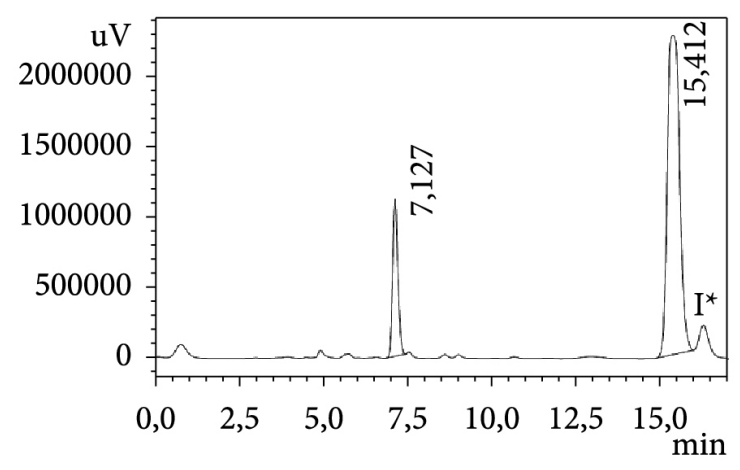

Figura 2 Cromatograma da piperlongumina no meio microssomal: $t_{R}=7,12$ minutos (produtos de oxidação), $t_{R}=15,41$ minutos (piperlongumina), I*: impureza da piperlongumina; condição cromatográfica: coluna Shim-pack VP-ODS Shimadzu ${ }^{\circledR}(250 \mathrm{~mm} \times 4,6 \mathrm{~mm}, 4,6 \mu \mathrm{m})$, vazão da

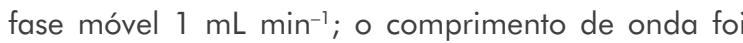
fixado em $325 \mathrm{~nm}$; volume injetado: $20 \mu \mathrm{L}$; fase móvel acetonitrila: água 40: 60, (v/v). 
mento de um método por CLUE-DAD. Apesar de na literatura haver descrições de softwares ou equações para conversão das condições entre as duas técnicas, preferiu-se elaborar uma a partir de várias tentativas e assim otimizar-se a separação dos compostos. A condição final foi: modo de eluição por gradiente com acetonitrila e água, ambas com $1 \%$ de ácido acético (v/v), que iniciava com $10 \%$ de ACN e atingia 100\% em 4 minutos. Conforme apresentado na Figura 3, há três picos, um relacionado à piperlongumina em $t_{R}=2,79$ min, o qual também apareceu na amostra controle, e, surpreendentemente, outros dois em $\mathrm{t}_{\mathrm{R}}=1,99 \min$ e $\mathrm{t}_{\mathrm{R}}=2,23$ min, relacionados aos produtos do metabolismo in vitro, os quais não apareceram na amostra controle, resultado diferente do apresentado anteriormente por CLAEDAD. O tempo para que ocorresse a separação foi inferior a três minutos e a análise total durou oito minutos (considerando o tempo de recondicionamento da coluna); muito inferior aos 18 minutos anteriormente relatados para CLAE, além de não haver perda de resolução entre os

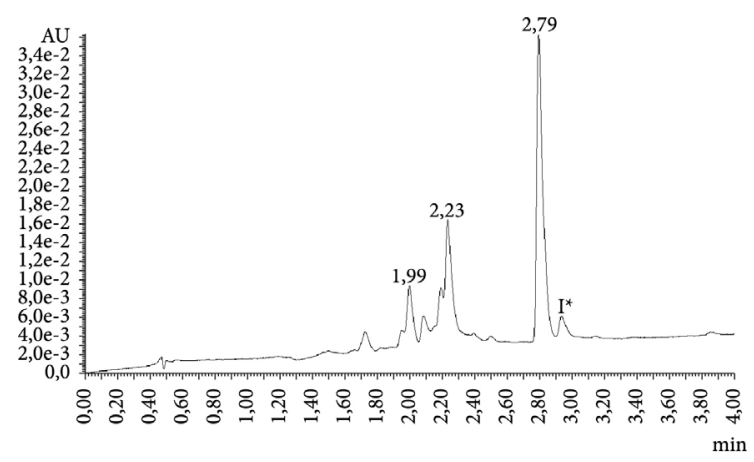

Figura 3 Cromatograma da piperlongumina no meio microssomal: $t_{R}=1,99$ minuto e $t_{R}=2,23$ minutos (produtos de oxidação), $t_{R}=2,79$ minutos (piperlongumina), I*: impureza da piperlongumina; condição cromatográfica: coluna Acquity ${ }^{\mathrm{TM}}$ UPLC BEH C18 (Bridged Ethylsiloxane Hybrid) (50 mm $\times$

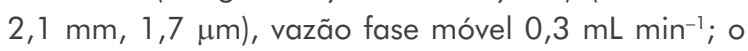
comprimento de onda foi fixado em $325 \mathrm{~nm}$; volume injetado: $5 \mu \mathrm{L}$; elvição por gradiente empregando acetonitrila e água, ambos com $1 \%$ de ácido acético $(\mathrm{v} / \mathrm{v})$, que iniciava com $10 \%$ de $\mathrm{ACN}$ e atingia $100 \%$ em 4 minutos. picos. Diferentemente do experimento com CLAE convencional, utilizou-se uma solução de concentração menor, $250 \mu \mathrm{g} \mathrm{mL}^{-1}$, sem a necessidade do pool das replicatas, porque os detectores ópticos em CLUE são projetados com volume da cela menor e um caminho óptico longo, o que mantém o sinal, causando baixa dispersão da amostra e obtendo-se alta detectabilidade ${ }^{[11,14]}$.

O exame desses resultados gerou uma dúvida: houve a formação de apenas um ou de dois metabólitos? Retornou-se à CLAE-DAD convencional para o desenvolvimento de outro método, dessa vez utilizando-se eluição por gradiente para, assim, comparar resultados com a CLUE. Após muitas tentativas, chegou-se à condição: acetonitrila e água, ambas com $1 \%$ de ácido acético (v/v), que iniciava com $10 \%$ de $\mathrm{ACN}$ e atingia 100\% em 30 minutos, a uma vazão de $1 \mathrm{~mL} \mathrm{~min}{ }^{-1}$. Conforme mostrado na Figura 4, há a presença de apenas dois picos, um relacionado à piperlongumina em $t_{R}=18,50$ min e outro em $t_{R}=14,80 \mathrm{~min}$, relacionado aos produtos do metabolismo in vitro. Os demais picos referem-se a interferentes graxos presentes na matriz.

Diante do exposto, chega-se a uma primeira hipótese de que realmente seriam formados dois produtos $^{[19]}$ oriundos de reação de oxidação, a qual é possível pelo sistema microssomal ${ }^{[21]}$. Tal reação os torna mais polares que a piperlongumina e, portanto, eles eluem em tempo anterior a ela. Outra conclusão foi a de que os compostos formados apresentam estruturas muito semelhantes, dado o comportamento cromatográfico idêntico no CLAE-DAD. Dessa forma, sugeriu-se que esses compostos tratavam-se de isômeros $^{[19]}$, pois a separação completa deles só foi conseguida com o emprego da CLUE, a qual possui um menor tamanho de partícula $(1,7 \mu \mathrm{m})$, elevado número de pratos e, portanto, uma maior eficiência. Dessa forma, o uso da CLUE foi fundamental nesse estudo, pois possibilitou uma maior separação dos analitos, ao contrário 


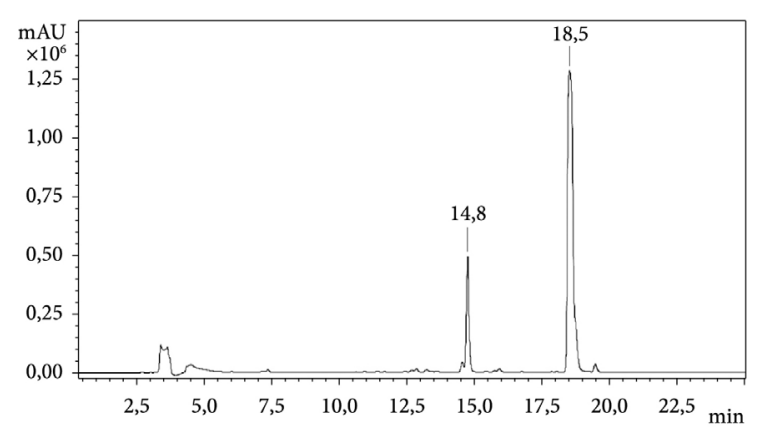

Figura 4 Cromatograma da piperlongumina no meio microssomal: $t_{\mathrm{Rv}}=14,80$ minutos (produtos de oxidação) e $t_{R}=18,50$ minutos (piperlongumina); condição cromatográfica: coluna Shim-pack VP-ODS Shimadzu ${ }^{\circledR}(250 \mathrm{~mm} \times 4,6 \mathrm{~mm}, 4,6 \mu \mathrm{m})$, vazão

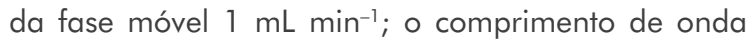
foi fixado em $325 \mathrm{~nm}$; volume injetado: $20 \mu \mathrm{L}$; fase móvel: acetonitrila e água, ambos com $1 \%$ de ácido acético (v/v), que iniciava com $10 \%$ de $A C N$ e atingia $100 \%$ em 30 minutos.

da CLAE convencional, que com emprego de uma coluna recheada com partículas de 4,6 $\mu \mathrm{m}$ de tamanho, não foi eficiente para separá-los no modo isocrático (coeluição) e nem no modo por gradiente (novamente coeluição), sendo que nesse último modo consumiram-se mais de 30 minutos na análise (levando-se em conta o tempo para o recondicionamento da coluna). Apesar dessa desvantagem, o emprego da CLAE convencional seria proveitoso para uma aplicação em escala preparativa, com a finalidade de isolamento dos compostos e sua caracterização.

Na hipótese de que seriam isômeros, desenvolveu-se um novo método, agora por cromatografia gasosa acoplada a espectrometria de massas, baseado no trabalho de Schaab et al. ${ }^{[22]}$. No ajuste da rampa de aquecimento, preferiu-se trabalhar com baixas taxas de aquecimento, mesmo que isso acarretasse maior tempo de análise, a fim de obter-se uma melhor capacidade de pico ${ }^{[23]}$, e optou-se pelo modo splitless, pois como os metabólitos estavam presentes em menor quantidade era necessário que toda a amostra fosse introduzida na coluna. Houve o aparecimento de três picos: um relacionado à piperlongumina em $t_{R}=38,99 \mathrm{~min}$, o qual também apareceu na amostra controle, e outros dois, em $t_{R}=39,93$ min e em $t_{R}=41,07$ min, relacionados aos produtos do metabolismo in vitro, os quais não apareceram na amostra controle ${ }^{[19]}$. Em todas as três técnicas não houve aparecimento de nenhum pico na amostra branco. Com isso confirma-se a hipótese de dois metabólitos e não apenas um. Por se tratar de produtos oxidados, há a formação de ligação de hidrogênio nas novas estruturas, que aumentam o ponto de fusão dos compostos e por isso eluem em tempo posterior à piperlongumina.

A técnica de cromatografia gasosa foi capaz de resolver todos os compostos presentes, contudo o tempo total requerido foi de 50 minutos e, por questões de detectabilidade (baixo volume de injeção e baixa capacidade da coluna) dos metabólitos, foi necessário injetar uma solução de concentração $1 \mathrm{mg} \mathrm{mL}^{-1}$, obtida a partir de um pool de 30 replicatas, devido à baixa concentração do substrato e, portanto, dos possíveis produtos. Esses dados confirmam a elevada eficiência apresentada pela CG devida, principalmente, ao longo comprimento da coluna cromatográfica, ao reduzido diâmetro da coluna e à rápida taxa de transferência de massa que ocorre entre a fase estacionária e o gás de arraste, levando, portanto, a uma maior eficiência na separação.

\section{Conclusão}

Conclui-se que as técnicas empregadas não se excluem e não são competitivas, pelo contrário, são complementares, cada uma com suas especificidades e limitações. É inevitável não destacar a economia de tempo e de solvente conseguida nas análises por CLUE, além da resolução e eficiência de separação entre picos com estruturas similares. Há que se levar em consideração a realidade de cada laboratório e a finalidade do analista em sua rotina quando se escolhe uma delas. 


\section{Agradecimentos}

Os autores gostariam de agradecer à FAPESP (Processos n' 2011/13560-9 e 2011/17508-1), ao CPNq e à CAPES pelo auxílio financeiro concedido.

\section{Referências}

1 Newman DJ, Cragg GM. Natural products as sources of new drugs over the 30 years from 1981 to 2010 . Journal of Natural Products 2012; 75:311-335. PMid:22316239 PMCid:PMC3721181. http://dx.doi. org/10.1021/np200906s

2 Marston A, Hostettmann K. Natural product analysis over the last decades. Planta Medica 2009; 75:672682. PMid:19263341. http://dx.doi. org/10.1055/s-0029-1185379

3 Gray MJ, Chang D, Zhang Y, Liu J, Bensoussan A. Development of liquid chromatography/mass spectrometry methods for the quantitative analysis of herbal medicine inbiological fluids: a review. Biomedical Chromatography 2010; 24:91-103. PMid:19650043. http://dx.doi.org/10.1002/bmc.1287

4 Wu H, Guo J, Chen S, Liu X, Zhou Y, Zhang $\mathrm{X}$, et al. Recent developments in qualitative and quantitative analysis of phytochemical constituents and their metabolites using liquid chromatographymass spectrometry. Journal of Pharmaceutical and Biomedical Analysis 2013; 72:267-291. PMid:23031576. http://dx.doi.org/10.1016/j.jpba.2012.09.004

5 Marston A. Role of advances in chromatographic techniques in phytochemistry. Phytochemistry 2007; 68:2786-2798. PMid:17931672. http://dx.doi.org/10.1016/j.phytochem.2007.08.004

6 MarriottPJ,ShellieR, CornwellC.Gaschromatographic technologies for the analysis of essential oils. Journal of Chromatography A 2001; 936:1-22. http://dx.doi. org/10.1016/S0021-9673(01)01314-0

7 Shikanga EA, Kamatou GPP, Chen W, Combrinck S, Viljoen AM. Validated RP-UHPLC PDA and GC-MS methods for the analysis of psychoactive alkaloids in Sceletium tortuosum. South African Journal of Botany 2012; 82:99-107. http://dx.doi.org/10.1016/j. sajb.2012.05.004

8 Choe S, Woo SH, Kim DW, Park Y, Choi H, Hwang BY, et al. Development of a target component extraction method from GC-MS data with an in-house program for metabolite profiling. Analytical
Biochemistry 2012; 426:94-102. PMid:22507375. http://dx.doi.org/10.1016/j.ab.2012.04.010

Wolfender JL. HPLC in natural product analysis: the detection issue. Planta Medica 2009; 75:719734. PMid:19145552. http://dx.doi. org/10.1055/s-0028-1088393

10 Baranowska I, Magiera S, Baranowski J. Clinical applications of fast liquid chromatography: A review on the analysis of cardiovascular drugs and their metabolite. Journal of Chromatography B 2013; 927:5479. PMid:23462623. http://dx.doi.org/10.1016/j. jchromb.2013.02.002

11 Swartz ME. UPLCTM: an introduction and review. Journal of Liquid Chromatography \& Related Technologies 2005; 28:1253-1263. http://dx.doi. org/10.1081/JLC-200053046

12 Avula B, Wang YH, Wang M, Smillie TJ, Khan IA. Simultaneous determination of sesquiterpenes and pyrrolizidine alkaloids from the rhizomes of Petasites hybridus (L.) G.M. et Sch. and dietary supplements using UPLC-UV and HPLC-TOF-MS methods. Journal of Pharmaceutical and Biomedical Analysis 2012; 70:53-63. PMid:22809670. http:// dx.doi.org/10.1016/j.jpba.2012.05.021

13 Funes NT, Serrano IO, Fusté JB, Moratalla MLL, Nogués MTV, Pulido MI, et al. Fast simultaneous determination of free and conjugated isoflavones in soy milk by UHPLC-UV. Food Chemistry 2012; 135:28322838. PMid:22980879. http://dx.doi.org/10.1016/j. foodchem.2012.06.011

14 Maldaner L, Jardim ICSF. O estado da arte da cromatografia líquida de ultra eficiência. Quimica Nova. 2009; 32:214-222. http://dx.doi.org/10.1590/ S0100-40422009000100036

15 Skraskova K, Santos LHMLM, Satinsky D, Pena A, Montenegro MCBSM, Solich P, et al. Fast and sensitive UHPLC methods with fluorescence and tandem mass spectrometry detection for the determination oftetracycline antibiotics in surface Waters. Journal of Chromatography B 2013; 927:201208. PMid:23340484. http://dx.doi.org/10.1016/j. jchromb.2012.12.032

16 James AT, Martin AJP. Gas-liquid partition chromatography: the separation and microestimation of volatile fatty acids from formic acid to dodecanoic acid. Biochemical Journal 1952; 50:679. PMid:14934673 PMCid:PMC1197726.

17 Bonato PS. Cromatografia gasosa. In: Collins C, Braga GL, Bonato PS. Fundamentos de Cromatografia. Campinas: Ed da Unicamp; 2006. p. 203-270. 
18 Tistaert C, Dejaegher B, Heyden YV. Chromatographic separation techniques and data handling methods for herbal fingerprints: A review. Analytica Chimica Acta 2011; 690:148-161. PMid:21435470. http:// dx.doi.org/10.1016/j.aca.2011.02.023

19 Marques LMM, Silva EA Jr, Gouvea DR, Vessecchi R, Pupo MT, Lopes NP, et al. In vitro metabolism of the alkaloid piplartine by rat liver microsomes. Journal of Pharmaceutical and Biomedical Analysis 2013. Subemtido a publicação.

20 Messiano GB, Santos RAS, Ferreira LS, Simões $\mathrm{RA}$, Jabor VAP, Kato MJ, et al. In vitro metabolism study of the promising anticancer agent the lignan (-)-grandisin. Journal of Pharmaceutical and Biomedical Analysis 2013; 72:240-244. PMid:22995290. http://dx.doi.org/10.1016/j.jpba.2012.08.028
21 Bourdon F, Lecoeur M, Verones V, Vaccher C, Lebegue $\mathrm{N}$, Dine $\mathrm{T}$, et al. In vitro pharmacokinetic profile of a benzopyridooxathiazepine derivative using rat microsomes and hepatocytes: Identification of phases I and II metabolites. Journal of Pharmaceutical and Biomedical Analysis 2013; 80:69-78. PMid:23528331. http://dx.doi.org/10.1016/j.jpba.2013.02.022

22 Schaab EH, Crotti AE, Iamamoto Y, Kato MJ, Lotufo LV, Lopes NP. Biomimetic oxidation of piperine and piplartine catalyzed by iron (III) and manganese (III) porphyrins. Biological \& Pharmaceutical Bulletin 2010; 33:912-916. http://dx.doi.org/10.1248/ bpb.33.912

23 Wang A, Tolley HD, Lee ML. Gas chromatography using resistive heating technology. Journal of Chromatography A 2012; 1261:46-57. PMid:22663978. http://dx.doi.org/10.1016/j.chroma.2012.05.021

\section{O FUTURO AGQRA É}

\section{SEPARADO DO PASSADO}

Imagine um laboratório onde todos os analistas podem obter dados de espectrometria de massas com alta qualidade. Por conta própria. Dentro da rotina existente. Para todas as amostras. Sem treinamento. Um laboratório onde a incerteza sobre compostos é substituída por confirmação rápida e eficiente obtida com a utilização de LC/MS nunca antes vista. Agora imagine isso tudo acontecendo com o apertar de um botão. Isso vai muito além do poder da detecção de massas. Isso é o ACQUITY QDa ${ }^{T M}$ Detector da Waters. SEPARATING BEYOND QUESTION ${ }^{\top M}$. Visite: waters.com/separate

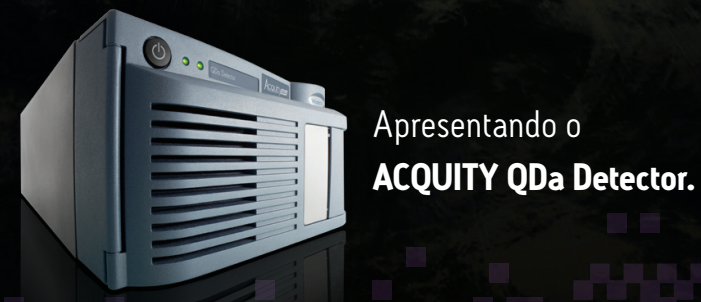

